\title{
Fluorographene as a Mass Spectrometry Probe for High-Throughput Identification and Screening of Emerging Chemical Contaminants in Complex Samples
}

\author{
Xiu Huang, ${ }^{\dagger \neq}$ Qian Liu, ${ }^{*},{ }^{\dagger} \S \odot$ Xiaoyu Huang, ${ }^{\| \odot}$ Zhou Nie, $^{\dagger}$ Ting Ruan, ${ }^{\dagger \odot}$ Yuguo Du, ${ }^{\dagger}$ and Guibin Jiang ${ }^{\dagger}$ \\ ${ }^{\dagger}$ State Key Laboratory of Environmental Chemistry and Ecotoxicology, Research Center for Eco-Environmental Sciences, Chinese \\ Academy of Sciences, Beijing 100085, China \\ ${ }^{\ddagger}$ State Key Laboratory of Chemo/Biosensing and Chemometrics, College of Chemistry and Chemical Engineering, Hunan University, \\ Changsha 410082, China \\ "Key Laboratory of Synthetic and Self-Assembly Chemistry for Organic Functional Molecules, Shanghai Institute of Organic \\ Chemistry, Chinese Academy of Sciences, Shanghai 200032, China \\ ${ }^{\S}$ Institute of Environment and Health, Jianghan University, Wuhan 430056, China
}

Supporting Information

\begin{abstract}
Mass spectrometry techniques for high-throughput analysis of complex samples are of profound importance in many areas such as food safety, omics studies, and environmental health science. Here we report the use of fluorographene (FG) as a new mass spectrometry probe for highthroughput identification and screening of emerging chemical contaminants in complex samples. FG was facilely synthesized by one-step exfoliation of fluorographite. With FG as a matrix or probe in matrix-assisted or surfaceenhanced laser desorption/ionization time-of-flight mass spectrometry (MALDI- or SELDI-TOF MS), higher sensitivity (detection limits at ppt or subppt levels), and better reproducibility were achieved than with other graphene-based materials due to the unique chemical structure and selfassembly properties of FG. The method was validated with different types of real complex samples. By using FG as a SELDI probe, we could easily detect trace amount of bisphenol $S$ in paper products and high-fat canned food samples. Furthermore, we have successfully identified and screened as many as 28 quaternary ammonium halides in sewage sludge samples collected from municipal wastewater treatment plants. These results demonstrate that FG probe is a powerful tool for high-throughput analysis of complex samples by MS.
\end{abstract}

$\mathrm{E}$ merging chemical contaminants (ECCs) are defined as chemicals that are not covered by standard monitoring and regulatory programs but may adversely affect the environment and human health. ${ }^{1-3}$ The list of ECCs is expanding with more and more chemicals' environmental occurrence and biological impacts being revealed. However, research into ECCs is limited by the lack of proper analytical methods. ${ }^{4}$ Due to their emerging nature, extremely low concentration (usually at parts per trillion to billion levels) and complex sample matrices, identification and quantification of ECCs in the environment is a great challenge. Current methods largely rely on chromatography coupled to mass spectrometry (MS), which however are laborious, low throughput, and require complicated sample preparation procedures. ${ }^{5}$

Matrix-assisted laser desorption/ionization time-of-flight mass spectrometry (MALDI-TOF MS) offers a highthroughput technique for analysis of large molecules. Surfaceenhanced laser desorption/ionization (SELDI) is a variant mode of MALDI that uses a probe for simultaneous extraction, enrichment, desorption, and ionization of target analytes. ${ }^{6-10}$

Recent exploration of new MALDI matrices and SELDI probes also extends their application to small molecule analysis. ${ }^{11-15}$ Specifically, graphene has shown great promise for the use as MALDI matrix or SELDI probe due to its excellent adsorption capacity, strong optical absorption properties, efficient electron-phonon coupling, and unique two-dimensional structure. $^{16-19}$ In practical applications, graphene usually needs to be chemically modified, such as oxidation ${ }^{20,21}$ and sulfonation, $^{22-25}$ to prevent its aggregation that may greatly compromise its performance in LDI MS. Graphene can also be used synergically with other materials or derivatized with functional groups to further improve the analytical sensitivity and selectivity. ${ }^{17,26}$

Fluorographene (FG) is an important derivative of graphene. ${ }^{27-30}$ Fluorination can tune the bandgap of graphene by converting the $\mathrm{C}-\mathrm{C}$ bonds from $\mathrm{sp}^{2}$ to $\mathrm{sp}^{3}$ configu-

Received: October 25, 2016

Accepted: December 13, 2016

Published: December 13, 2016 


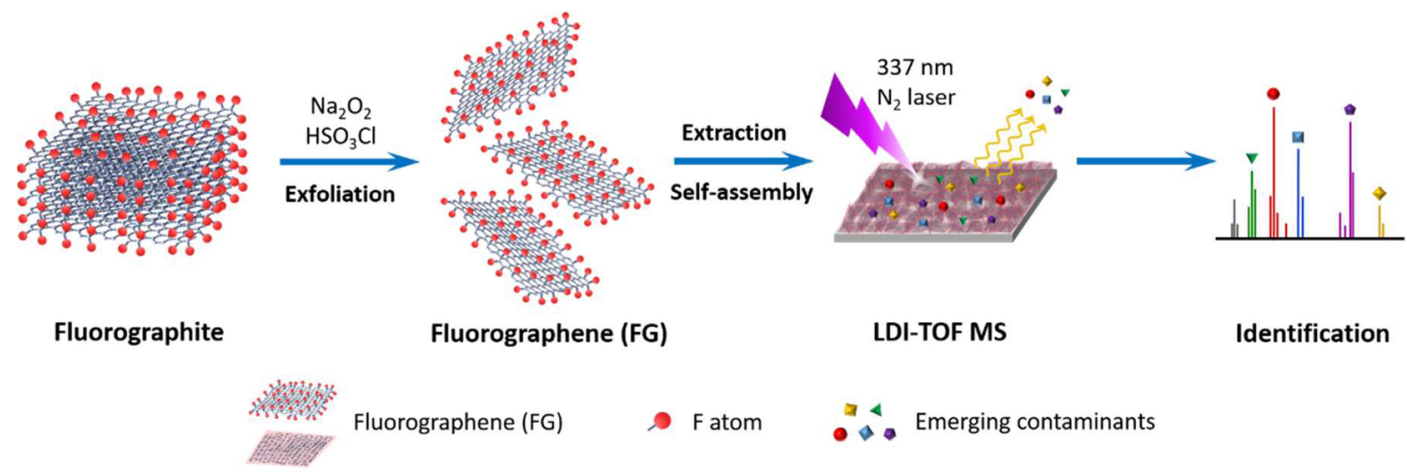

Figure 1. Scheme showing the procedures for the identification and screening of ECCs in complex samples by SELDI-TOF MS with FG as a probe.

ration. ${ }^{31,32}$ Compared with pure graphene, the introduction of $\mathrm{F}$ atoms may offer additional opportunity for FG to form hydrogen bonding with target compounds due to the high electronegativity of $\mathrm{F}$ atoms. ${ }^{33,34}$ Furthermore, theoretical calculations have predicted that FG is more thermodynamically stable than other graphene derivatives, ${ }^{35}$ suggesting that FG may produce fewer fragments and less background noises in the LDI process. Therefore, FG may serve as a good SELDI probe for a wide variety of analytes. However, to the best of our knowledge, the application of FG in LDI MS is so far unexplored.

Inspired by the intriguing properties of FG, we here show the use of FG as a new SELDI probe for high-throughput identification and screening of ECCs in complex samples. We found that FG could self-assemble into a honeycomb-like film and showed better performance than other graphene-based materials in MALDI- and SELDI-TOF MS. High sensitivity and good reproducibility were obtained with the FG probe. More importantly, the method was validated with different types of real complex samples. By using FG as a SELDI probe, we could detect trace amount of bisphenol S (BPS) in thermal receipt paper and canned food samples. We have also identified and screened over 20 quaternary ammonium halides in sewage sludge samples collected from municipal wastewater treatment plants. These results demonstrate that FG is a powerful tool for MS screening of small molecules in extremely complex samples.

\section{EXPERIMENTAL SECTION}

Chemicals and Materials. Chemically converted graphene and graphene oxide (GO) were bought from XFNANO (Nanjing, China). Fluorographite (F\% > 56\%) was purchased from CarFluor Chemicals (Shanghai, China). BPS, tetradecyldimethylbenzylammonium chloride hydrate (TDBAC), and tetrabromobisphenol A (TBBPA) were from TCI (Tokyo, Japan). 2,2',4,4'-Tetrabromodiphenyl ether (BDE-47) was from AccuStandard (New Haven, CT, U.S.A.). Arsenic(III) standard solution at $1000 \mu \mathrm{g} / \mathrm{mL}$ in $0.5 \%$ (v/v) $\mathrm{HCl} / 0.3 \%$, (w/ v) $\mathrm{NaOH} / 0.06 \%,(\mathrm{w} / \mathrm{v}) \mathrm{NaHCO}_{3}$ solution was purchased from Inorganic Ventures (Christiansburg, VA). Estradiol (E2) and pentachlorophenol (PCP) were from Dr. Ehrenstorfer (Augsburg, Germany). Hexadecyldimethylbenzylammonium chloride (HDBAC), dodecyldimethylbenzylammonium chloride (DDBAC), cetyltrimethylammonium bromide (CTAB), tetradecyltrimethylammonium bromide (TTAB), perfluorooctanesulfonate (PFOS), and $\alpha$-cyano-4-hydroxycinnamic acid (CHCA) were from Sigma (St. Louis, MO). Dichloromethane and acetone of HPLC grade were from J.T. Baker (Phillipsburg, $\mathrm{NJ})$. Chlorosulfonic acid $\left(\mathrm{HSO}_{3} \mathrm{Cl}, 99.0 \%\right)$ and sodium peroxide $\left(\mathrm{Na}_{2} \mathrm{O}_{2}, 95 \%\right)$ were from SinoPharm (Shanghai, China). N-Methyl-2-pyrrolidone (NMP, 99.0\%) was from Alfa Aesar (Ward Hill, MA). Ultrapure water was prepared by a Millipore Milli-Q system (Billerica, MA). All chemicals were of analytical grade unless otherwise noted.

Synthesis of FG. FG was synthesized by exfoliating fluorographite with sodium peroxide and chlorosulfonic acid as described previously. ${ }^{36}$ First of all, $0.1 \mathrm{~g}$ of fluorographite powder and $1 \mathrm{~g}$ of $\mathrm{Na}_{2} \mathrm{O}_{2}$ were added to a corundum crucible and ground evenly. Then, $5 \mathrm{~mL}$ of $\mathrm{HSO}_{3} \mathrm{Cl}$ was added dropwise to the mixture under stirring. After cooling down at room temperature, $100 \mathrm{~mL}$ of water was added to dilute the mixture. The obtained solution was filtered through a $0.22 \mu \mathrm{m}$ poly(vinylidene fluoride) membrane. The solids were collected and washed with water three times followed by drying at $60{ }^{\circ} \mathrm{C}$ overnight. Finally, a black powder of FG was obtained. Note: the reaction of $\mathrm{Na}_{2} \mathrm{O}_{2}$ with $\mathrm{HSO}_{3} \mathrm{Cl}$ is violent and this step of operation should be carried out very carefully.

Characterization of Materials. SEM images were obtained by using a Hitachi S-3000N scanning electron microscope (Tokyo, Japan). FTIR spectra were obtained on a JASCO FT/IR Fourier transform infrared spectrometer (Victoria, B.C., Canada). The samples were pressed into transparent disks with $\mathrm{KBr}$ for the measurement. XPS spectra were obtained using a Thermo Scientific Escalab 250Xi X-ray photoelectron spectrometer (Massachusetts, U.S.A.) with $\mathrm{Al}$ $\mathrm{K} \alpha \mathrm{X}$-ray radiation as the $\mathrm{X}$-ray source excitation.

MALDI- and SELDI-TOF MS. For direct MALDI-TOF MS measurement, the sample solution and the matrix dispersion were mixed at a ratio of $1: 1(\mathrm{v} / \mathrm{v})$, and then $1 \mu \mathrm{L}$ of the mixture was dropped to a stainless steel MTP target frame III (Bruker Daltonics) followed by air drying.

For SELDI-TOF MS measurement (see Figure 1), the FG probe was dispersed in water at $10 \mathrm{mg} / \mathrm{mL}$ under ultrasonication. Then, $500 \mu \mathrm{L}$ of the probe was added to $2 \mathrm{~mL}$ of sample solution to enrich target analytes. The solution was incubated at room temperature for $48 \mathrm{~h}$. The probe was then collected by centrifugation at $9000 \mathrm{rpm}$ for $15 \mathrm{~min}$. The supernatant was discarded and the precipitate $(2 \mu \mathrm{L})$ was deposited on the MALDI target for analysis.

The LDI-TOF MS was performed on a Bruker Daltonics Autoflex III Smartbean MALDI-TOF mass spectrometer equipped with a $337 \mathrm{~nm}$ nitrogen laser working at a frequency of $100 \mathrm{~Hz}$ and controlled by the FlexControl software. The measurement was performed in reflector mode. The mass spectra were recorded by summing 200 laser shots. The FlexAnalysis 3.4 software was used for data processing. 
Analysis of Real Samples. The thermal receipt paper samples were collected from supermarkets, grocery stores, and restaurants in Beijing $(n=9)$. The spam and canned sardines samples were bought from three supermarkets in Beijing. Each sample was homogenized and extracted with $15 \mathrm{~mL}$ of ultrapure water by the aid of sonication for $90 \mathrm{~min}$. The mixture was centrifuged at $9000 \mathrm{rpm}$ for $10 \mathrm{~min}$ and the supernatant was used for the analysis.

The sewage sludge samples were collected during Oct 2010 to May 2011 from 12 municipal wastewater treatment plants in 6 provinces and municipalities in China. Approximately $500 \mathrm{~g}$ wet weight of the freshly digested sludge sample obtained from the dehydration process was freeze-dried, homogenized, and stored at $-20{ }^{\circ} \mathrm{C}$. Then, $0.2 \mathrm{~g}$ of solid sample was extracted with $8 \mathrm{~mL}$ of ultrapure water under sonication for $90 \mathrm{~min}$. The sample was centrifuged at $9000 \mathrm{rpm}$ for $10 \mathrm{~min}$, and then the supernatant was subjected to the SELDI-TOF MS analysis.

\section{RESULTS AND DISCUSSION}

Characterization of FG. Before testing the performance in MS, we characterized the materials by different techniques. FG was synthesized by one-step exfoliation of fluorographite. ${ }^{36}$ The as-synthesized FG showed a good water-dispersibility. SEM measurement showed that the FG could form a homogeneous and flat film on the MALDI target (Figure 2A). Interestingly, we found that when the aqueous dispersion of FG was incubated exceeding $48 \mathrm{~h}$ before being dropped to the MALDI target, the formed FG film could turn into a honeycomb-like three-dimensional (3D) structure (Figure 2B). The reason was ascribed to the self-assembly of FG sheets driven by strong

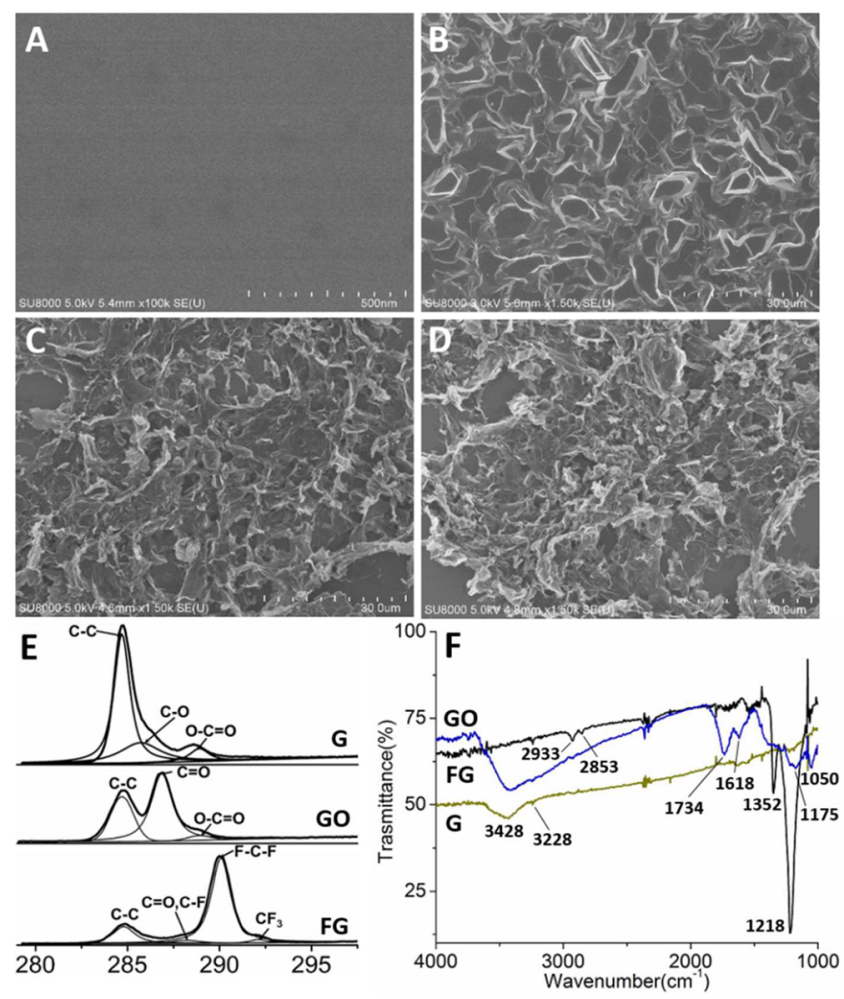

Figure 2. Characterization of materials. (A-D) Typical SEM images of FG film after incubation for $1 \mathrm{~h}$ (A), FG film after incubation for 48 $\mathrm{h}(\mathrm{B})$, graphene film after incubation for $1 \mathrm{~h}(\mathrm{C})$, and graphene film after incubation for $48 \mathrm{~h}(\mathrm{D})$. (E) C 1s XPS spectra of graphene (G), GO, and FG. (F) FT-IR spectra of G, GO, and FG. hydrophobic interaction and hydrogen bonding. ${ }^{37}$ Theoretical studies have predicted that fluorination may transform the $\mathrm{sp}^{2}$ bonding planar crystal structure of graphene into a $\mathrm{sp}^{3}$-bonding 3D structure. ${ }^{37,38}$ The phenomenon observed here provides an evidence for this prediction. Such a 3D structure was not observed when the incubation time was $1 \mathrm{~h}$ (Figure 2A), indicating that the self-assembly of FG was a relatively slow process. For comparison, pure graphene formed an inhomogeneous and rugged film on the MALDI target, leaving a considerable part of the MALDI target surface being exposed (Figure 2C). When prolonging the incubation time from 1 to $48 \mathrm{~h}$, the graphene film tended to aggregate due to its strong hydrophobic nature rather than forming any ordered structures (Figure 2D), suggesting that the $\mathrm{F}$ atoms played a critical role in the self-assembly of FG.

Figure 2E compares the C 1s XPS spectra of graphene, GO, and FG. FG yielded four main components at 284.8, 288.1, 290.3, and $292.2 \mathrm{eV}$, assigning to $\mathrm{C}-\mathrm{C}, \mathrm{C}-\mathrm{F}, \mathrm{F}-\mathrm{C}-\mathrm{F}$, and $\mathrm{CF}_{3}$ bonds, respectively. The strong peak of $\mathrm{F}-\mathrm{C}-\mathrm{F}$ bond indicates a high degree of fluorination in FG. The peaks of oxygen functionalities for FG were much lower than those for GO and graphene, indicating that $\mathrm{C}-\mathrm{F}$ bonds were the major functionality in FG. EDX measurement showed that the ratio of $\mathrm{C}$ to $\mathrm{F}$ atoms in FG was 1.1:1 (Supporting Information, Figure S1), indicating that the FG was nearly fully fluorinated. The materials were also characterized by FTIR (Figure 2F). FG exhibited a sharp peak at 1218 and $1352 \mathrm{~cm}^{-1}$, which are ascribed to the feature absorption of the $\mathrm{C}-\mathrm{F}$ and $\mathrm{F}-\mathrm{C}-\mathrm{F}$ stretch. $^{31}$ The peaks at 2933 and $2853 \mathrm{~cm}^{-1}$ are assigned to the asymmetric and symmetric $\mathrm{C}-\mathrm{H}$ stretching vibration, respectively. ${ }^{39}$ Graphene and GO had a broad peak at $\sim 3428 \mathrm{~cm}^{-1}$ due to the $\mathrm{O}-\mathrm{H}$ stretching vibration and showed no $\mathrm{C}-\mathrm{F}$ absorption peaks. The peaks at 1734,1618 , and $1050 \mathrm{~cm}^{-1}$ are assigned to the $\mathrm{C}=\mathrm{O}$ stretching vibration, the skeletal ring vibrations, or the stretching vibration of the $\mathrm{C}-\mathrm{O}$ bond. ${ }^{39}$ Solid absorption spectra showed that the optical absorption of FG at $337 \mathrm{~nm}$ (the wavelength used in MALDI) was close to GO but less than graphene (Figure S2).

Use of FG as a MALDI Matrix. In order to be used as a SELDI probe, the material must be able to serve as an efficient MALDI matrix. So we first investigated the performance of FG as a MALDI matrix. A variety of chemical contaminants with molecular weight below $1000 \mathrm{Da}$ were used as model analytes (see Table 1), including two flame retardants (TBBPA and BDE-47), a synthetic fluorosurfactant (PFOS), an endocrine disrupter (E2), an organochlorine pesticide (PCP), an

Table 1. Feature Peaks and LODs $(\mathrm{S} / \mathrm{N}=3)$ of Typical Chemical Contaminants Obtained with FG as a SELDI Probe

\begin{tabular}{llllllll}
\multicolumn{3}{c}{ negative ion } & & \multicolumn{3}{c}{ positive ion } \\
\cline { 1 - 4 } \cline { 5 - 7 } compound & $m / z$ & $\begin{array}{c}\mathrm{LOD}^{a} \\
(\mathrm{pg} / \mathrm{mL})\end{array}$ & & compound & $m / z$ & $\begin{array}{c}\mathrm{LOD}^{a} \\
(\mathrm{pg} / \mathrm{mL})\end{array}$ \\
BPS & 248.4 & 0.7 & & As $s^{\mathrm{II}}$ & 152.4 & $1.5 \times 10^{3}$ \\
BDE-47 & 250.2 & 0.03 & & TTAB & 255.9 & 0.07 \\
PCP & 264.2 & 0.06 & & CTAB & 284.0 & 0.07 \\
E2 & 270.5 & 38 & & DDBAC & 304.0 & 3.8 \\
PFOS & 498.5 & $6 \times 10^{-3}$ & & TDBAC & 332.1 & 0.4 \\
TBBPA & 542.4 & 0.6 & & HDBAC & 360.1 & $5.0 \times 10^{2}$
\end{tabular}

${ }^{a}$ The LODs were obtained based on the highest feature peaks of the analytes. 
industrial adhesive (BPS), two linear surfactants (CTAB and TTAB), three aromatic surfactants (HDBAC, TDBAC, and DDBAC), and inorganic As ${ }^{\mathrm{III}}$. These chemicals have been widely used in industrial and consumer products but pose potential risks to human health and ecosystems (see section 1.1 in Supporting Information for details).

The performance of different materials (including FG, graphene, GO, and conventional organic matrix $\mathrm{CHCA}$ ) as MALDI matrices was compared in detection of these typical contaminants. As shown in Figure 3A,B, when using FG as a

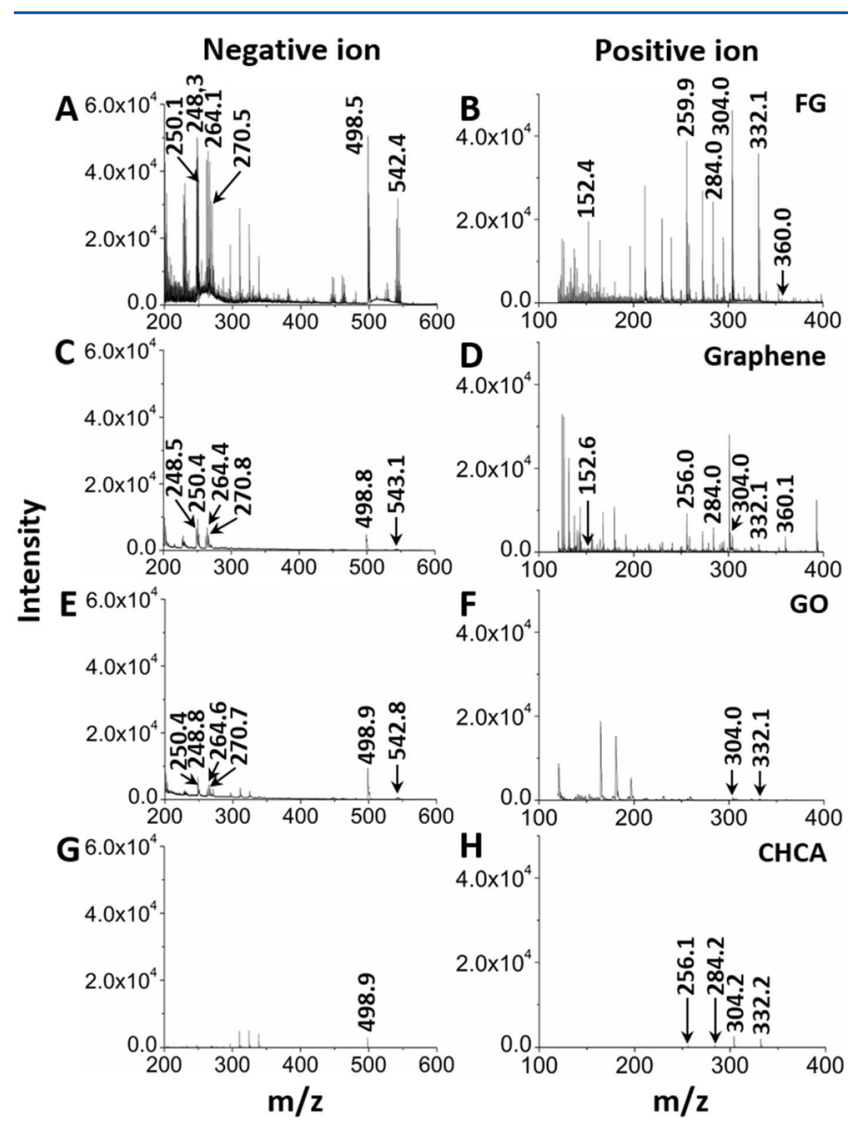

Figure 3. Comparison of performance of different materials as matrices in MALDI-TOF MS detection of typical ECCs (BPS, BDE47, PCP, E2, PFOS, TBBPA, As ${ }^{\mathrm{III}}$, HDBAC, TDBAC, DDBAC, CTAB, and TTAB): (A, B) FG, (C, D) graphene, (E, F) GO, and (G, $\mathrm{H}) \mathrm{CHCA}$. The left and right column represent the spectra obtained in negative and positive ion mode, respectively. The samples were incubated with the matrix for $48 \mathrm{~h}$ before the analysis. Analyte concentration: BPS, $10 \mu \mathrm{g} / \mathrm{mL}$; BDE- $47,5 \mu \mathrm{g} / \mathrm{mL}$; PCP, $10 \mu \mathrm{g} / \mathrm{mL}$; E2, $50 \mu \mathrm{g} / \mathrm{mL}$; PFOS, $1 \mu \mathrm{g} / \mathrm{mL}$; TBBPA, $10 \mu \mathrm{g} / \mathrm{mL}$; As ${ }^{\text {III }}, 15 \mu \mathrm{g} / \mathrm{mL}$; HDBAC, $5 \mu \mathrm{g} / \mathrm{mL}$; TDBAC, $5 \mu \mathrm{g} / \mathrm{mL}$; DDBAC, $5 \mu \mathrm{g} / \mathrm{mL}$; CTAB, 1 $\mu \mathrm{g} / \mathrm{mL}$; and TTAB, $1 \mu \mathrm{g} / \mathrm{mL}$.

matrix, all compounds could be readily detected by MALDITOF MS with strong peak intensities. Notably, we found that the incubation time of matrix dispersion with sample solution significantly affected the MS signals. With an incubation time of $1 \mathrm{~h}$, the peak intensities with FG were relatively low (Figure S3). When increasing the incubation time to $48 \mathrm{~h}$, the peak intensities significantly increased and FG showed the best performance in both negative and positive ion mode (Figure 3). Such a remarkable increase in MS signals could be well explained by the structural transformation of the FG film. As shown in Figure 2A,B, FG could self-assemble to form a honeycomb-like structure upon increasing the incubation time. This structure not only could increase the surface area of the film for laser energy absorption and transfer, but also effectively avoided the aggregation of FG sheets. In contrast, the peak intensities obtained with graphene and GO decreased with the increase in the incubation time (Figures 3 and S3) probably due to the aggregation of the materials. It should be noted that the graphene matrix incubated for $1 \mathrm{~h}$ could also generate high peak intensities in negative ion mode, but in positive ion mode the peak intensities were lower than those obtained with the FG matrix incubated for $48 \mathrm{~h}$ (Figure S3). For CHCA (Figure $3 \mathrm{G}, \mathrm{H})$, only weak peaks of $[\mathrm{M}-\mathrm{H}]^{-}$of PFOS in negative ion mode, $[\mathrm{M}-\mathrm{Br}]^{+}$of $\mathrm{CTAB}$ and TTAB, and $[\mathrm{M}-\mathrm{Cl}]^{+}$of DDBAC and TDBAC in positive ion mode were detected, confirming that conventional organic matrices were not suitable for analysis of small molecules.

The fluorination of graphene also greatly affected the reproducibility of analysis. MALDI-TOF MS often suffers from the reproducibility due to the inhomogeneous cocrystallization process of the matrix with analytes. With a short incubation time $(1 \mathrm{~h})$, the shot-to-shot $(n=20)$ and sample-tosample RSDs $(n=15)$ obtained on the FG matrix were in the ranges of $22.2-95.1 \%$ and $38.0-91.7 \%$, respectively (Table $\mathrm{S} 1$ ). When the incubation time was increased to $48 \mathrm{~h}$, the shotto-shot and sample-to-sample RSDs were significantly improved to $12.6-29.4 \%$ and $14.5-32.3 \%$, respectively (Table S2). This reproducibility was satisfactory for MALDITOF MS analysis and also better than that obtained on graphene and GO matrices, e.g., the shot-to-shot and sampleto-sample RSDs on the graphene matrix were in the ranges of $34.6-90.6 \%$ and $36.6-91.4 \%$, respectively (Tables S1 and S2). The good reproducibility of FG should result from the relatively stable and uniform structure of the FG film. The limits of detection (LODs) for the typical contaminants by direct MALDI-TOF MS measurement on the FG matrix ranged from 0.5 to $\left(7.5 \times 10^{5}\right) \mathrm{pg} / \mathrm{mL}$ (Table S3), which were lower than those obtained with graphene and GO (Table S3). Overall, FG has been demonstrated to be a highly efficient matrix for MALDI-TOF MS.

Use of FG as a SELDI Probe. After demonstration of the capability of FG as a MALDI matrix, we further attempted to use FG as a SELDI probe. The probe was added to sample solution to enrich target compounds, and then the probe was collected and directly subjected to LDI-TOF MS analysis. We first tested the method with spiked water samples with spiking concentrations at $\mathrm{ppb}$ levels and compared the performance of FG with graphene and GO. As shown in Figure 4A,B, all 12 typical contaminants could be detected by using the FG probe and the peak intensities were significantly higher than those using other materials. With graphene as a probe (Figure 4C,D), BPS, PCP, PFOS, BDE-47, and E2 could be detected in negative ion mode, and TTAB and TDBAC could be detected in positive ion mode. However, their peak intensities were low and the peaks for other compounds were absent. When using GO as a probe (Figure 4E,F), only PFOS was detected in negative ion mode. These results support our hypothesis that FG is a good SELDI probe due to its special fluorinated structure, which can enhance the affinity of FG for a wide range of compounds via hydrophobic interaction and hydrogen bonding.

Table 1 lists the LODs for the typical contaminants obtained by SELDI-TOF MS with the FG probe. The corresponding mass spectra are given in Figure S4. For most of compounds 


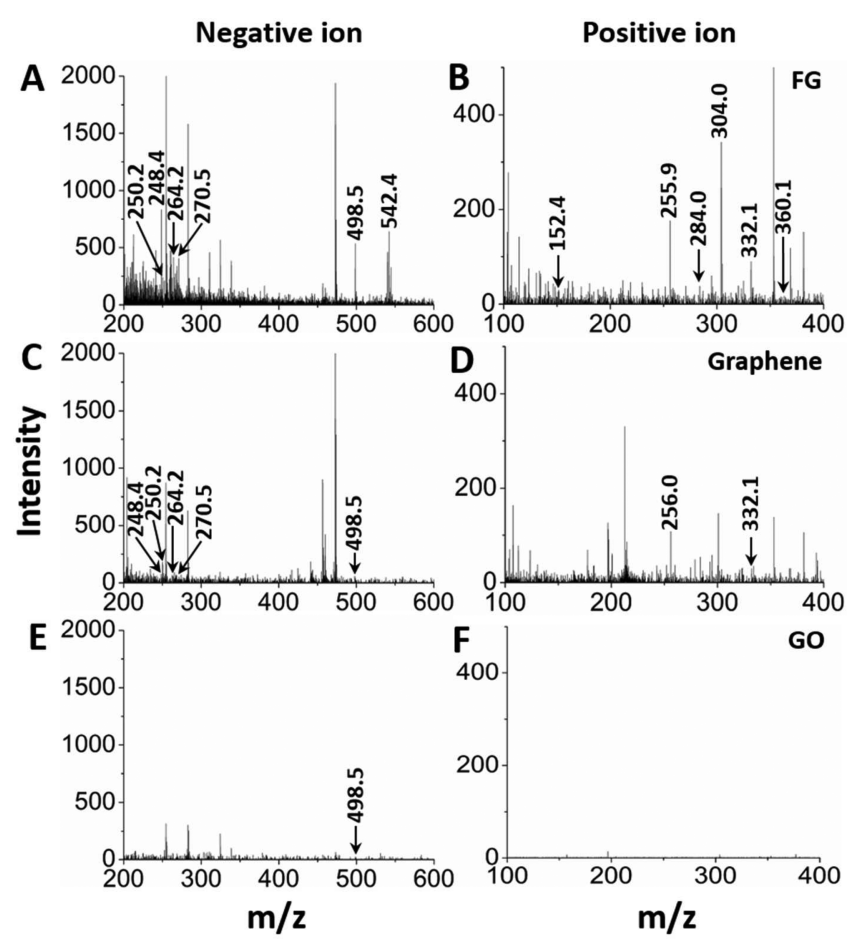

Figure 4. Comparison of performance of different materials as SELDI probes in detection of typical ECCs (BPS, BDE-47, PCP, E2, PFOS, TBBPA, As $\left.{ }^{\mathrm{III}}, \mathrm{HDBAC}, \mathrm{TDBAC}, \mathrm{DDBAC}, \mathrm{CTAB}, \mathrm{TTAB}\right)$ in spiked water samples. The left and right column represent the spectra obtained in negative and positive ion mode, respectively. (A, B) FG, (C, D) graphene, (E, F) GO. Spike concentration: TBBPA, $6.6 \mathrm{ng} /$ $\mathrm{mL}$; PFOS, $0.66 \mathrm{ng} / \mathrm{mL}$; E2, $33.1 \mathrm{ng} / \mathrm{mL}$; PCP, $6.6 \mathrm{ng} / \mathrm{mL}$; BDE-47, $3.3 \mathrm{ng} / \mathrm{mL}$; and BPS, $6.6 \mathrm{ng} / \mathrm{mL}$; As ${ }^{\mathrm{III}}, 9.9 \mathrm{ng} / \mathrm{mL}$; HDBAC, $3.3 \mathrm{ng} /$ $\mathrm{mL}$; TDBAC, $3.3 \mathrm{ng} / \mathrm{mL}$; DDBAC, $3.3 \mathrm{ng} / \mathrm{mL}$; CTAB, $0.66 \mathrm{ng} / \mathrm{mL}$; and TTAB, $0.66 \mathrm{ng} / \mathrm{mL}$.

(except for $\mathrm{As}{ }^{\mathrm{III}}$ ), the LODs were at ppt or subppt levels (0.006-500 pg/mL). These LODs can satisfy the demands for routine screening of these contaminants. For As ${ }^{\mathrm{III}}$, an additional enrichment procedure may be required to meet the "action level" of $10 \mathrm{ppb}$ set by the U.S. EPA. ${ }^{40}$ Comparing the LODs obtained by SELDI- and MALDI-TOF MS (Tables 1 and S3), the LODs were significantly lowered by $7.1-\left(8.3 \times 10^{5}\right)$-fold by the FG enrichment (except for HDBAC). It is worth mentioning that the LOD for PFOS was low to $6 \mathrm{fg} / \mathrm{mL}$, probably because PFOS is also a fully fluorinated compound, and thus, FG has a particularly strong affinity for PFOS. We also compared the present method with previously reported methods such as HPLC or GC coupled to MS in terms of LOD. For most of compounds (except for As ${ }^{\mathrm{III}}$ and HDBAC), the present method yielded lower or comparable LODs than previously reported methods (see Table S4).

Screening of BPS in Real Complex Samples. To demonstrate the application potential in analysis of complex samples, we used the FG probe to screen BPS in different types of real samples. As a substitute of bisphenol A (BPA), BPS is widely used as fixing and anticorrosive agents in epoxy glues, but it has also been identified as a potential endocrine disruptor and immunotoxicant. ${ }^{41}$ Recently, the occurrence of BPS in paper products and foods has been reported. ${ }^{42-44}$ So in this study we selected thermal receipt papers, spams, and canned sardines as target sample matrices. The analysis of food samples like spams and canned sardines is normally regarded as a very challenging task due to their high fat contents, and complicated sample preparation procedures are required to analyze these samples.

Here the FG probe was used to detect BPS at ppt levels in thermal receipt papers, spams, and canned sardines. Direct analysis of these samples by MALDI-TOF MS without enrichment produced no signals corresponding to BPS due to its low concentrations present in these samples (Figure S5). When using FG as a SELDI probe, peak of $[\mathrm{M}-\mathrm{H}]^{-}$of BPS was detected at $m / z 248.8$ in all samples with few interferences caused by sample matrices (Figure 5 ). The concentration was

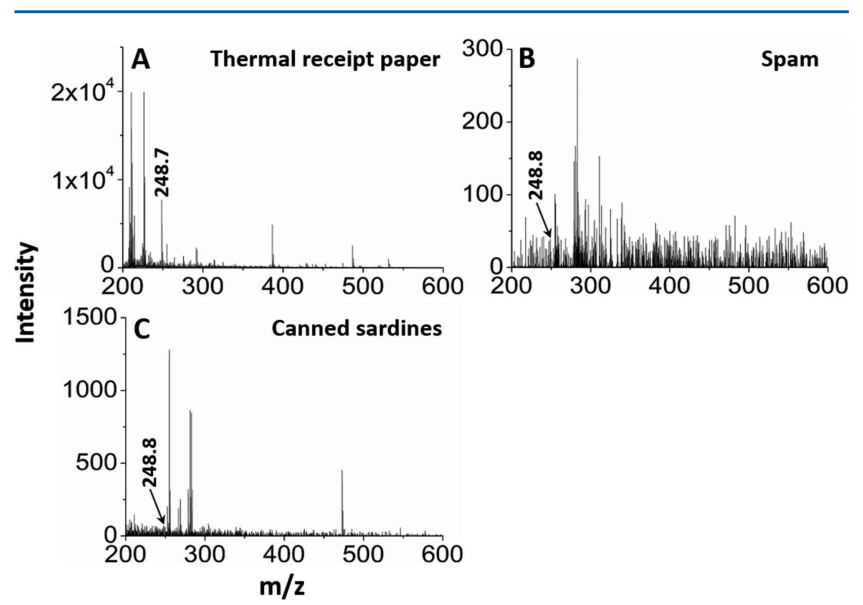

Figure 5. Screening of BPS in real complex samples by SELDI-TOF MS with FG as a probe: (A) thermal receipt paper samples, (B) spam samples, and $(\mathrm{C})$ canned sardines samples.

determined by the standard addition method $\left(R^{2}>0.988\right.$; see Table S5). High concentration of BPS was found in thermal receipt paper samples $(1.4-2.8 \mu \mathrm{g} / \mathrm{g} ; n=9)$, which was within the range that reported in previous reports (Table S6). ${ }^{42,43}$ Based on the obtained concentration, the daily intake of BPS is estimated to be $0.08-0.16 \mathrm{ng} /$ day for the generation population and 6.1-12.2 ng/day for occupationally exposed individuals (see section 1.2 in Supporting Information for estimation method). The BPS was also detected in canned food samples at lower concentrations $(0.011-0.17 \mu \mathrm{g} / \mathrm{g}$ for spam $(n$ $=3$ ) and $0.0015-0.048 \mu \mathrm{g} / \mathrm{g}$ for canned sardines $(n=3)$; see Table S5). These results demonstrate that FG is a sensitive probe for screening of trace amount of ECCs in high-fat complex samples.

Identification and Screening of ECCs in Sewage Sludge Samples. We then applied the FG probe in identification and screening of ECCs in sewage sludge samples collected from 12 municipal wastewater treatment plants. The sewage sludge is considered as a major sink of urban contaminants and is recycled by the application of biosolids. ${ }^{45-48}$ In this study, we focused on quaternary ammonium halides as target ECCs. Quaternary ammonium halides, as a class of cationic surfactants, are widely used in commercial products. They have shown potential persistency in the environment ${ }^{49,50}$ and can affect the bioavailability and mobility of hydrophobic organic contaminants. ${ }^{51}$ The SELDI-TOF MS analysis was carried out in positive ion mode. In control experiments, no ECCs were identified in blank water samples (Figure S6). In sewage sludge samples, a large number of peaks were detected from $\mathrm{m} / \mathrm{z} 200$ to 610 (Figure 6A). Among them, we have successfully identified the feature peaks of as many as 28 quaternary ammonium halides (Figure 6A). This number 

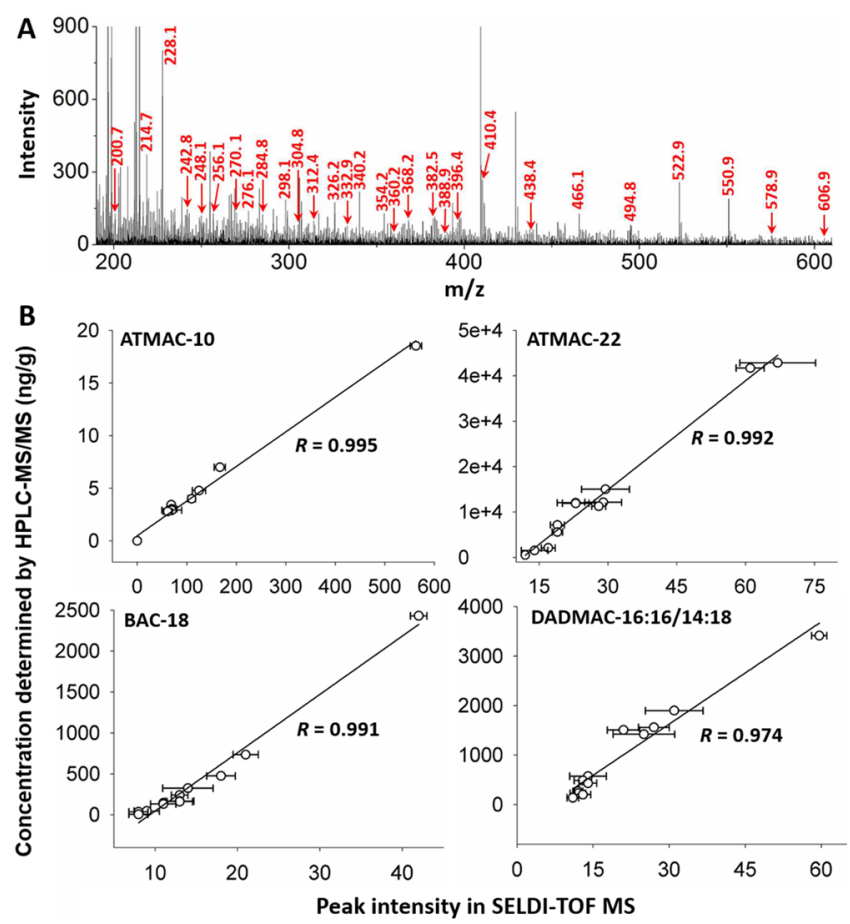

Figure 6. Identification and screening of quaternary ammonium halides in municipal sewage sludge samples collected from 12 municipal wastewater treatment plants. (A) A typical mass spectrum of sewage sludge samples. (B) Correlation of analytical results of ATMAC-10, ATMAC-22, BAC-18, and DADMAC-16:16/14:18 obtained by SELDI-TOF MS with concentrations determined by HPLC-ESI-MS/MS. Note that the lines in (B) just show the comparison results between the two methods but are not calibration curves for the compounds.

was the same as that obtained in a previous work using HPLCESI-MS/MS. ${ }^{47}$ The names, molecular formulas, and feature peaks of the identified compounds are listed in Tables 2 and S7. For comparison, we have also analyzed the sewage sludge samples by using graphene as a SELDI probe with an incubation time of $1 \mathrm{~h}$, but only two types of quaternary ammonium halides could be identified (see Figure S7).
To verify the results obtained by the FG-based SELDI-TOF MS, we also measured the concentrations of the quaternary ammonium halides in sewage sludge samples by HPLC-ESIMS/MS (see section 1.3 in Supporting Information for experimental details). Excellent linear correlations $(R>$ 0.929) was found between the peak intensities of quaternary ammonium halides in SELDI-TOF MS and the concentrations obtained by HPLC-ESI-MS/MS. Figure $6 \mathrm{~B}$ shows the results for ATMAC-10, ATMAC-22, BAC-18, and DADMAC-16:16/ $14: 18$ as examples $(R>0.974)$. Data for other quaternary ammonium halides are given in Figure S8. The good consistency between these two techniques proves the accuracy of the identification results and demonstrates that SELDI-TOF MS is a reliable method for identification and screening of ECCs in complex samples. The comparison results also show that quaternary ammonium halides are present in sewage sludge at relatively high concentrations, e.g., the concentration of DADMAC-18:18 could reach $157 \mu \mathrm{g} / \mathrm{g}$ (Figure 6B). This calls for more attention paid to these compounds present in the environment. Finally, it should be stressed that sewage sludge is a highly complex matrix. The conventional analytical methods involves complicated and laborious sample preparation procedures. ${ }^{47}$ As an alternative, the FG-based SELDI-TOF MS technique provides a much more facile, high-throughput, and sensitive tool for the analysis of low-mass compounds in complex samples.

\section{CONCLUSIONS}

In summary, we have shown that FG can be used as a new MALDI matrix and SELDI probe for identification and screening of ECCs in complex samples. The FG was facilely prepared using a one-step exfoliation method from fluorographite. In LDI MS, FG could form a honeycomb-like film by self-assembly, which could facilitate the MS detection. Using FG as a MALDI matrix or SELDI probe, higher sensitivity and better reproducibility than other graphene-based materials were obtained. The method was successfully applied to identify and screen ECCs in different types of real complex samples, including thermal receipt paper, canned foods, and sewage sludge samples. This work not only provides a new promising tool for high-throughput identification and screening of lowmass compounds, but also further fulfils the capability of LDI

Table 2. Acronyms, Molecular Formulas, and Feature Peaks of the Identified Quaternary Ammonium Halides in Sewage Sludge Samples by Using the FG Probe ${ }^{a}$

\begin{tabular}{|c|c|c|c|c|c|}
\hline compound & molecular formula & $m / z$ & compound & molecular formula & $m / z$ \\
\hline ATMAC-10 & $\mathrm{C}_{13} \mathrm{H}_{30} \mathrm{NBr}$ & 200.7 & DADMAC-8:8 & $\mathrm{C}_{18} \mathrm{H}_{40} \mathrm{NBr}$ & 270.1 \\
\hline ATMAC-12 & $\mathrm{C}_{15} \mathrm{H}_{34} \mathrm{NBr}$ & 228.1 & DADMAC-8:10 & $\mathrm{C}_{20} \mathrm{H}_{44} \mathrm{NBr}$ & 298.1 \\
\hline ATMAC-14 & $\mathrm{C}_{17} \mathrm{H}_{38} \mathrm{NBr}$ & 256.1 & DADMAC-10:10 & $\mathrm{C}_{22} \mathrm{H}_{48} \mathrm{NBr}$ & 326.2 \\
\hline ATMAC-16 & $\mathrm{C}_{19} \mathrm{H}_{42} \mathrm{NBr}$ & 284.8 & DADMAC-12:12 & $\mathrm{C}_{26} \mathrm{H}_{56} \mathrm{NBr}$ & $382.5 ; 214.7$ \\
\hline ATMAC-18 & $\mathrm{C}_{21} \mathrm{H}_{46} \mathrm{NBr}$ & 312.4 & DADMAC-14:14 & $\mathrm{C}_{30} \mathrm{H}_{64} \mathrm{NBr}$ & $438.4 ; 242.8$ \\
\hline ATMAC-20 & $\mathrm{C}_{23} \mathrm{H}_{50} \mathrm{NBr}$ & 340.2 & DADMAC-16:16 & $\mathrm{C}_{34} \mathrm{H}_{72} \mathrm{NBr}$ & 494.8 \\
\hline ATMAC-22 & $\mathrm{C}_{25} \mathrm{H}_{54} \mathrm{NCl}$ & 368.2 & DADMAC-18:18 & $\mathrm{C}_{38} \mathrm{H}_{80} \mathrm{NBr}$ & 550.9 \\
\hline ATMAC-24 & $\mathrm{C}_{27} \mathrm{H}_{58} \mathrm{NCl}$ & 396.4 & DADMAC-20:20 & $\mathrm{C}_{42} \mathrm{H}_{88} \mathrm{NBr}$ & 606.9 \\
\hline BAC-8 & $\mathrm{C}_{17} \mathrm{H}_{30} \mathrm{NCl}$ & 248.1 & DADMAC-10:12 & $\mathrm{C}_{24} \mathrm{H}_{52} \mathrm{NBr}$ & 354.2 \\
\hline BAC-10 & $\mathrm{C}_{19} \mathrm{H}_{34} \mathrm{NCl}$ & 276.1 & DADMAC-12:14 & $\mathrm{C}_{28} \mathrm{H}_{60} \mathrm{NBr}$ & 410.4 \\
\hline BAC- 12 & $\mathrm{C}_{21} \mathrm{H}_{38} \mathrm{NCl}$ & 304.8 & DADMAC-14:16 & $\mathrm{C}_{32} \mathrm{H}_{68} \mathrm{NBr}$ & 466.1 \\
\hline BAC-14 & $\mathrm{C}_{23} \mathrm{H}_{42} \mathrm{NCl}$ & 332.9 & DADMAC-14:18 & $\mathrm{C}_{34} \mathrm{H}_{72} \mathrm{NBr}$ & 494.8 \\
\hline BAC-16 & $\mathrm{C}_{25} \mathrm{H}_{46} \mathrm{NCl}$ & 360.2 & DADMAC-16:18 & $\mathrm{C}_{36} \mathrm{H}_{76} \mathrm{NBr}$ & 522.9 \\
\hline BAC-18 & $\mathrm{C}_{27} \mathrm{H}_{50} \mathrm{NCl}$ & 388.9 & DADMAC-18:20 & $\mathrm{C}_{40} \mathrm{H}_{84} \mathrm{NBr}$ & 578.9 \\
\hline
\end{tabular}

${ }^{a_{T}}$ The full names and chemical structures are given in Table S7. 
MS in small molecule analysis by extending its application to more complex sample matrices than before. Future efforts will be made to understand whether other functionalities of graphene (e.g., sulfonation) can bring new benefits in the MS analysis.

\section{ASSOCIATED CONTENT}

\section{S Supporting Information}

The Supporting Information is available free of charge on the ACS Publications website at DOI: 10.1021/acs.analchem.6b04167.

Supporting experimental details, supporting tables, supporting figures, and supporting references (PDF).

\section{AUTHOR INFORMATION}

\section{Corresponding Author}

*E-mail: qianliu@rcees.ac.cn.

\section{ORCID}

Qian Liu: 0000-0001-8525-7961

Xiaoyu Huang: 0000-0002-9781-972X

Ting Ruan: 0000-0002-6222-374X

\section{Notes}

The authors declare no competing financial interest.

\section{ACKNOWLEDGMENTS}

The authors acknowledge financial support from the Chinese Academy of Sciences (Nos. XDB14010400, QYZDB-SSWDQC018), the National Basic Research Program of China (2015CB931903, 2015CB932003), and the National Natural Science Foundation of China (Nos. 21377141, 21422509, 91543104). Q.L. also acknowledges the support from the Youth Innovation Promotion Association of CAS.

\section{REFERENCES}

(1) Daughton, C. G. Renew. Resour. J. 2005, 23, 6.

(2) la Farre, M.; Perez, S.; Kantiani, L.; Barcelo, D. TrAC, Trends Anal. Chem. 2008, 27, 991-1007.

(3) Deblonde, T.; Cossu-Leguille, C.; Hartemann, P. Int. J. Hyg. Environ. Health 2011, 214, 442-448.

(4) Liu, Q.; Zhou, Q.; Jiang, G. TrAC, Trends Anal. Chem. 2014, 58, $10-22$.

(5) Richardson, S. D. Anal. Chem. 2012, 84, 747-778.

(6) Liu, Z.; Yuan, Z.; Zhao, Q. PLoS One 2014, 9, el03276.

(7) Tang, N.; Tornatore, P.; Weinberger, S. R. Mass Spectrom. Rev. 2004, 23, 34-44.

(8) Merchant, M.; Weinberger, S. R. Electrophoresis 2000, 21, 11641177.

(9) Grizzle, W. E.; Semmes, O. J.; Bigbee, W.; Zhu, L.; Malik, G.; Oelschlager, D. K.; Manne, B.; Manne, U. Cancer Inform. 2005, 1, 8697.

(10) Issaq, H. J.; Veenstra, T. D.; Conrads, T. P.; Felschow, D. Biochem. Biophys. Res. Commun. 2002, 292, 587-592.

(11) Wang, J.; Liu, Q.; Liang, Y.; Jiang, G. Anal. Bioanal. Chem. 2016, 408, 2861-2873.

(12) Wang, J.; Liu, Q.; Gao, Y.; Wang, Y.; Guo, L.; Jiang, G. Anal. Chem. 2015, 87, 6931-6936.

(13) Chen, L.; Ou, J.; Wang, H.; Liu, Z.; Ye, M.; Zou, H. ACS Appl. Mater. Interfaces 2016, 8, 20292-20300.

(14) Zhao, Y.; Deng, G.; Liu, X.; Sun, L.; Li, H.; Cheng, Q.; Xi, K.; $\mathrm{Xu}$, D. Anal. Chim. Acta 2016, 937, 87-95.

(15) Huang, X.; Liu, Q.; Fu, J.; Nie, Z.; Gao, K.; Jiang, G. Anal. Chem. 2016, 88, 4107-4113.
(16) Favre-Kontula, L.; Sattonnet-Roche, P.; Magnenat, E.; Proudfoot, A. E. I.; Boschert, U.; Xenarios, L.; Vilbois, F.; Antonsson, B. Proteomics 2008, 8, 378-388.

(17) Gulbakan, B.; Yasun, E.; Shukoor, M. I.; Zhu, Z.; You, M.; Tan, X.; Sanchez, H.; Powell, D. H.; Dai, H.; Tan, W. J. Am. Chem. Soc. 2010, 132, 17408-17410.

(18) Tang, L. A. L.; Wang, J.; Loh, K. P. J. Am. Chem. Soc. 2010, 132, 10976-10977.

(19) Dong, X.; Cheng, J.; Li, J.; Wang, Y. Anal. Chem. 2010, 82, 6208-6214.

(20) Liu, Q.; Cheng, M.; Jiang, G. Chem. - Eur. J. 2013, 19, 55615565.

(21) Liu, Q.; Cheng, M.; Wang, J.; Jiang, G. Chem. - Eur. J. 2015, 21, 5594-5599.

(22) Liu, Y.-M.; Shi, G.-F.; Zhang, J.-J.; Zhou, M.; Cao, J.-T.; Huang, K.-J.; Ren, S.-W. Colloids Surf., B 2014, 122, 287-293.

(23) Shi, C.; Meng, J.; Deng, C. Chem. Commun. 2012, 48, $2418-$ 2420.

(24) Min, Q.; Zhang, X.; Chen, X.; Li, S.; Zhu, J.-J. Anal. Chem. 2014, $86,9122-9130$

(25) Sha, Y.; Huang, D.; Zheng, S.; Deng, C. Anal. Methods 2013, 5, $4585-4590$

(26) Wang, J.; Cheng, M.; Zhang, Z.; Guo, L.; Liu, Q.; Jiang, G. Chem. Commun. 2015, 51, 4619-4622.

(27) Nair, R. R.; Ren, W.; Jalil, R.; Riaz, I.; Kravets, V. G.; Britnell, L.; Blake, P.; Schedin, F.; Mayorov, A. S.; Yuan, S.; Katsnelson, M. I.; Cheng, H.-M.; Strupinski, W.; Bulusheva, L. G.; Okotrub, A. V.; Grigorieva, I. V.; Grigorenko, A. N.; Novoselov, K. S.; Geim, A. K. Small 2010, 6, 2877-2884.

(28) Hong, X.; Cheng, S. H.; Herding, C.; Zhu, J. Phys. Rev. B: Condens. Matter Mater. Phys. 2011, 83, n/a.

(29) Robinson, J. T.; Burgess, J. S.; Junkermeier, C. E.; Badescu, S. C.; Reinecke, T. L.; Perkins, F. K.; Zalalutdniov, M. K.; Baldwin, J. W.; Culbertson, J. C.; Sheehan, P. E.; Snow, E. S. Nano Lett. 2010, 10, 3001-3005

(30) Jeon, K.-J.; Lee, Z.; Pollak, E.; Moreschini, L.; Bostwick, A.; Park, C.-M.; Mendelsberg, R.; Radmilovic, V.; Kostecki, R.; Richardson, T. J.; Rotenberg, E. ACS Nano 2011, 5, 1042-1046.

(31) Zboril, R.; Karlicky, F.; Bourlinos, A. B.; Steriotis, T. A.; Stubos, A. K.; Georgakilas, V.; Safarova, K.; Jancik, D.; Trapalis, C.; Otyepka, M. Small 2010, 6, 2885-2891.

(32) Wang, Z.; Wang, J.; Li, Z.; Gong, P.; Liu, X.; Zhang, L.; Ren, J.; Wang, H.; Yang, S. Carbon 2012, 50, 5403-5410.

(33) Howard, J. A. K.; Hoy, V. J.; O'Hagan, D.; Smith, G. T. Tetrahedron 1996, 52, 12613-12622.

(34) Espinosa, E.; Alkorta, I.; Elguero, J.; Molins, E. J. Chem. Phys. 2002, 117, 5529-5542.

(35) Wang, L.-F.; Ma, T.-B.; Hu, Y.-Z.; Wang, H.; Shao, T.-M. J. Phys. Chem. C 2013, 117, 12520-12525.

(36) Yang, Y.; Lu, G.; Li, Y.; Liu, Z.; Huang, X. ACS Appl. Mater Interfaces 2013, 5, 13478-13483.

(37) Li, Y.; Li, F.; Chen, Z. J. Am. Chem. Soc. 2012, 134, 1126911275 .

(38) Withers, F.; Dubois, M.; Savchenko, A. K. Phys. Rev. B: Condens. Matter Mater. Phys. 2010, 82, 073403.

(39) Iwamoto, R.; Ohta, K. Appl. Spectrosc. 1984, 38, 359-365.

(40) Supporting Document for Action Level for Arsenic in Apple Juice; U.S. Food and Drug Administration: Silver Spring, MD, U.S.A., 2013.

(41) Vinas, R.; Watson, C. S. Environ. Health 2013, 12.

(42) Liao, C. Y.; Liu, F.; Kannan, K. Environ. Sci. Technol. 2012, 46, $6515-6522$

(43) Vinas, P.; Campillo, N.; Martinez-Castillo, N.; HernandezCordoba, M. Anal. Bioanal. Chem. 2010, 397, 115-125.

(44) Liao, C. Y.; Kannan, K. J. Agric. Food Chem. 2013, 61, 46554662.

(45) Cirelli, A. F.; Ojeda, C.; Castro, M. J. L.; Salgot, M. Environ. Chem. Lett. 2008, 6, 135-148.

(46) Clarke, B. O.; Smith, S. R. Environ. Int. 2011, 37, 226-247. 
(47) Ruan, T.; Song, S. J.; Wang, T.; Liu, R. Z.; Lin, Y. F.; Jiang, G. B. Environ. Sci. Technol. 2014, 48, 4289-4297.

(48) Song, S. J.; Song, M. Y.; Zeng, L. Z.; Wang, T.; Liu, R. Z.; Ruan, T.; Jiang, G. B. Environ. Pollut. 2014, 186, 14-19.

(49) Lara-Martin, P. A.; Li, X. L.; Bopp, R. F.; Brownawell, B. J. Environ. Sci. Technol. 2010, 44, 7569-7575.

(50) Martinez-Carballo, E.; Gonzalez-Barreiro, C.; Sitka, A.; Kreuzinger, N.; Scharf, S.; Gans, O. Environ. Pollut. 2007, 146, 543547.

(51) Sanchez, L.; Romero, E.; Sanchez-Rasero, F. S.; Dios, G.; Pena, A. Pest Manage. Sci. 2003, 59, 857-864. 